\title{
Synthesis and characterization of stable dicarboxylic pegylated magnetite nanoparticles
}

\author{
Sara Gil ${ }^{\mathrm{a}, \mathrm{b}}$, Emilio Castro ${ }^{\mathrm{a}, \mathrm{b}}$, João F. Mano ${ }^{\mathrm{a}, \mathrm{b}, *}$ \\ a 3B's Research Group-Biomaterials, Biodegradables and Biomimetics, University of Minho, Headquarters of the European Institute of Excellence on Tissue Engineering and \\ Regenerative Medicine, AvePark, 4806-909 Taipas, Guimarães, Portugal \\ b ICVS/3B's-PT Government Associate Laboratory, Braga/Guimarães, Portugal
}

\section{A R T I C L E I N F O}

Article history:

Received 20 December 2012

Accepted 11 March 2013

Available online 20 March 2013

Keywords:

Magnetic nanoparticles

MRI

Nanomedicine

Superparamagnetism

Biomaterials

\begin{abstract}
A B S T R A C T
The coating of implantable nano- or micro-objects with polyethylene glycol (PEG) enhances its biocompatibility and biodistribution. Herein, we describe a new protocol that enhances and maintains MNPs stability in biological media, simulating multiple conditions to which they would be subjected in the human body. Magnetite nanoparticles (MNPs) prepared via a facile way at room temperature by coprecipitation reaction, were coated with dicarboxylic polyethylene glycol (DCPEG) via covalent bonds. The surface of the nanoparticles was first coated with 3-aminopropyl trimethoxysilane by a silanization reaction and then linked with DCPEG of different molecular weight $(M w=5000,10,000$ and $20,000 \mathrm{~g} \mathrm{~mol}^{-1}$ ). The uncoated magnetite nanoparticles, with an average size of $20 \mathrm{~nm}$, exhibited superparamagnetism, high saturation magnetization and a negative surface charge (with a zeta potential value of $-40 \mathrm{mV}$ ). Increase of $\mathrm{Mw}$ enhances the colloidal stability of MNPs and makes them more suitable to tolerate high salt concentrations $(1 \mathrm{M} \mathrm{NaCl})$ and wide $\mathrm{pH}$ (from 5.5 to 12 ) and temperature ranges $\left(24^{\circ} \mathrm{C}\right.$ to $\left.46^{\circ} \mathrm{C}\right)$. The results indicate that magnetite nanoparticles coated with DCPEG with $M w=20,000$ have improved properties over their counterparts, making them our best choice for biomedical studies.
\end{abstract}

(c) 2013 Elsevier B.V. All rights reserved.

\section{Introduction}

The stability of magnetic nanoparticles (MNPs) in aqueous media results from the equilibrium established between attractive and repulsive forces. Attractive forces come from van der Waals and magnetic dipolar attractions, while repulsive forces mainly originate from electrostatic and steric repulsions [1]. In aqueous solutions, the Fe atoms coordinate with water, which dissociates readily to leave the iron oxide surface hydroxyl functionalized. These hydroxyl groups are amphoteric and may react with acids and bases [2]. Depending upon the $\mathrm{pH}$ of the solution, the surface of the magnetite will be positively or negatively charged. For superparamagnetic iron oxide nanoparticles (SPIONs) to be stable in aqueous media at physiological $\mathrm{pH}$ (7.35-7.45 for human blood), it is necessary to bring in additional charges to the nanoparticle surface. This is very important for electrostatically stabilizing the colloids due to the fact that the isoelectric point (IEP) of "naked" SPIONs is observed at $\mathrm{pH} 6.8$ [3]. Around this point of zero charge

\footnotetext{
*Corresponding author at: 3B's Research Group-Biomaterials, Biodegradables and Biomimetics, University of Minho, Headquarters of the European Institute of Excellence on Tissue Engineering and Regenerative Medicine, AvePark, 4806-909 c, Guimarães, Portugal

E-mail address: jmano@dep.uminho.pt (J.F. Mano).
}

(PZC), the surface charge density is too small and the particles are no longer stable in water and flocculate. Matching both electrostatic and steric stabilization, allows to obtain stable SPIONs [4-6].

Nevertheless, satisfying colloidal stability around neutral $\mathrm{pH}$ is not enough for magnetic nanoparticles to be useful as magneticfield-directed drug targeting and as contrast agents for magnetic resonance imaging administrated intravenously; they should also have good enough colloidal stability at physiological ionic strength around $0.17 \mathrm{M}$. Phosphate buffered saline (PBS) is often used to mimic the $\mathrm{pH}$ and ionic strength of physiological conditions as the osmolarity and ion concentrations of the PBS buffer match those of the human body [1]. However, in biological media, electrostatically stable MNPs are prone to aggregation due to neutralization of the surface charge [7]. Therefore, polymers are often used as stabilizing agents as they provide steric repulsion to the MNPs in addition to electrostatic repulsion, thus being able to reduce the influence of ionic strength on the colloidal stability.

Under physiological conditions, the effective minimization of SPIONs aggregation, caused by protein adsorption, must also be taken into account. Thus, anti-biofouling polymers are preferred to modify the MNPs for producing reticuloendothelial system evading nanoparticles with long blood half-lifes [8]. Among antibiofouling polymers, polyethylene glycol (PEG) is one of the good 
choices. It has been demonstrated that PEG coated $\mathrm{Fe}_{3} \mathrm{O}_{4}$ nanoparticles present long blood circulation time [9].

Dicarboxyl-terminated PEG (HOOC-PEG-COOH), DCPEG, was adopted as the nanoparticle surface capping agent for providing the magnetic nanoparticle biocompatibility, meanwhile offering free surface carboxylic acid groups for further covalently conjugating the $\mathrm{Fe}_{3} \mathrm{O}_{4}$ nanoparticles to bioligands bearing amine groups, via the EDC/NHS mediated amidation reaction [10-14]. It has been demonstrated that $\mathrm{Fe}_{3} \mathrm{O}_{4}$ nanoparticles coated by dicarboxylterminated poly(ethylene glycol) (HOOC-PEG-COOH) can be used for constructing MRI dual modality molecular probes for in vivo colorectal carcinoma and gastric carcinoma detection $[10,15,16]$. Herein, we study the important effect of the molecular weight of DCPEG on the colloidal stability of magnetite nanoparticles, and present a new surface engineering approach that produces a highly stable polymeric coating and MNPs.

\section{Experimental section}

Preparation of DCPEG-Fe $\mathrm{O}_{3} \mathrm{O} M \mathrm{NPS}$ : All the reagents used in this work were analytical grade without further purification. Iron (III) chloride hexahydrate $\left(\mathrm{FeCl}_{3} \cdot 6 \mathrm{H}_{2} \mathrm{O}, \geq 99 \%\right)$, iron (II) chloride tetrahydrate $\left(\mathrm{FeCl}_{2} \cdot 4 \mathrm{H}_{2} \mathrm{O}, 99 \%\right)$, ammonium hydroxide $\left(\mathrm{NH}_{4} \mathrm{OH}, 33 \%\right.$ $\mathrm{NH}_{3}$ in $\left.\mathrm{H}_{2} \mathrm{O}\right)$ were obtained from Sigma. DCPEG $5000(M w \approx$ $4900 \mathrm{~g} \mathrm{~mol}^{-1}$-Figure S1 of Supporting Information), DCPEG 10000 $\left(M w \approx 11,400 \mathrm{~g} \mathrm{~mol}^{-1}\right.$-Figure $\mathrm{S} 2$ of Supporting Information), $\mathrm{DCPEG}_{20000}\left(M w \approx 20,000 \mathrm{~g} \mathrm{~mol}^{-1}\right)$ were all purchased from Nano OcS. (3-aminopropyl) triethoxysilane (APTES) was a coupling agent from Sigma. N-(3-dimethylaminopropyl)-N'-ethylcarbodiimide hydrochloride (EDC, $\geq 98 \%$ ) and $\mathrm{N}$-hydroxysuccinimide (NHS) were bought from Sigma. In all experiments, deionized water was used.

The procedure used for the preparation of MNPs and coating by silanization reaction was based on a previously described protocol [17]. The magnetite $\left(\mathrm{Fe}_{3} \mathrm{O}_{4}\right)$ nanoparticles were synthesized by the co-precipitation reaction of ferrous and ferric salts with $\mathrm{NH}_{4} \mathrm{OH}$ acting as a reducing agent. Then the MNPs were coated with APTES and finally linked with dicarboxyl-terminated DCPEG via the EDC/NHS reaction between $-\mathrm{NH}_{2}$ and $-\mathrm{COOH}$ to form welldispersed surface functionalized biocompatible MNPs.

Characterization: The morphology of $\mathrm{Fe}_{3} \mathrm{O}_{4}$ and DCPEG- $\mathrm{Fe}_{3} \mathrm{O}_{4}$ was observed using a Philips CM-12 transmission electron microscope (TEM) operating at an acceleration voltage of $100 \mathrm{kV}$. Samples for TEM were obtained by the evaporation of a drop of the solution sample $\left(C=1 \mathrm{mg} \mathrm{mL}^{-1}\right)$ on a carbon-coated copper grid (400 mesh), under ambient conditions. Later the images were analyzed by Image $J$ software (NIH). The dynamic light scattering (DLS) and zeta $(\zeta)$ potential measurements were performed with a Nano-ZS, Malvern Zetasizer. Ultrasonic dispersion was performed for $15 \mathrm{~min}$ for each sample before DLS and $\zeta$ potential measurements. All measurements were repeated three times. Magnetic properties were measured on a vibrating sample magnetometer (VSM) (PPMS, Quantum Design) at $25^{\circ} \mathrm{C}$ in a field up to $100 \mathrm{kOe}$. Mass spectroscopy measurements (MALDI-TOF) were carried out using a ULTRAFLEX III TOF/TOF (BRUKER).

\section{Results and discussion}

Fig. 1A presents the average size of MNPs coupled with DCPEG with $M w$ of $5000,10,000$ and $20,000 \mathrm{~g} \mathrm{~mol}^{-1}$, in aqueous medium obtained by DLS. Before modification the MNPs exhibit an average
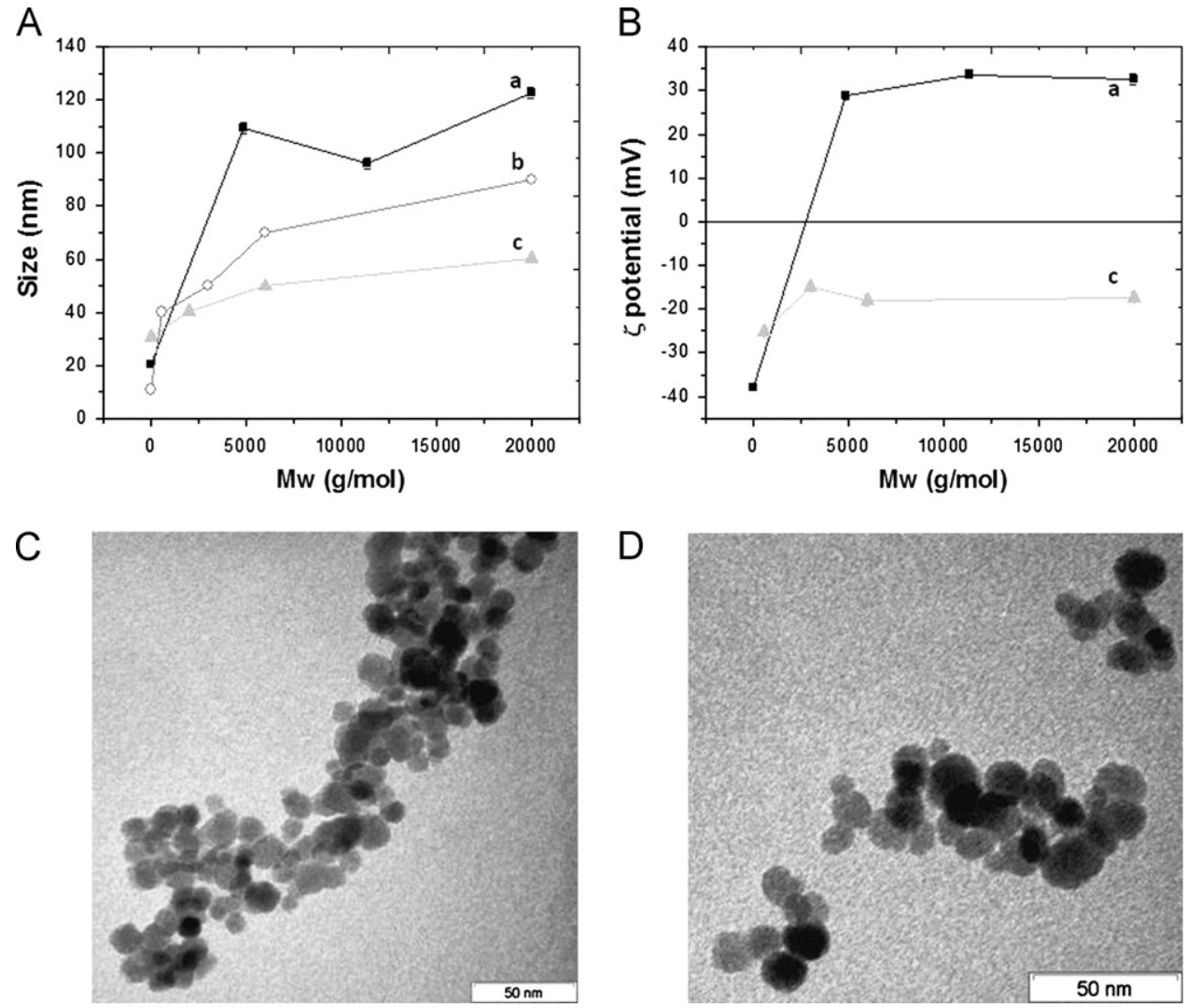

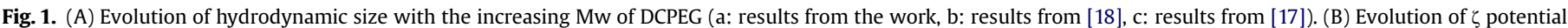
with the increasing Mw of DCPEG (a: results from the work, c: results from [18]). TEM images of (C) $\mathrm{Fe}_{3} \mathrm{O}_{4}$ and (D) DCPEG-Fe $\mathrm{O}_{4}$. 
hydrodynamic diameter of $20 \mathrm{~nm}$. After modification the MNPs' size increases with the increasing length of DCPEG chains from $110 \mathrm{~nm}$ up to around $120 \mathrm{~nm}$. Both Xie et al. [18] and Feng et al. [17] also found a similar behavior-see comparison in Fig. 1A. $\zeta$ potential measurements show that before the modification the MNPs are stable $(|\zeta| \geq 25 \mathrm{mV})$ and negatively charged. However, when coated with DCPEG, the MNPs exhibit a positively charged $\zeta$ potential that remains positive regardless of DCPEG Mw (Fig. 1B). The positive $\zeta$ potential can be explained by the hydration of polymer coating surrounding the magnetite core, which increases steric repulsion. Another factor is that not all the $-\mathrm{NH}_{2}$ groups in APTES react with DCPEG and, in culture medium, these groups can exist in a cationic form.

TEM images show that both $\mathrm{Fe}_{3} \mathrm{O}_{4}$ (Fig. 1C) and DCPEG- $\mathrm{Fe}_{3} \mathrm{O}_{4}$ (Fig. 1D) MNPs present spherical shape and a core size of $14.8 \pm 2.9 \mathrm{~nm}$ and $13.8 \pm 3.4 \mathrm{~nm}$, respectively. The low contrast of the organic coating explains the similarity of these values.

For clinical purposes it is important that DCPEG- $\mathrm{Fe}_{3} \mathrm{O}_{4} \mathrm{MNPs}$ maintain the same magnetic properties as before the modification. Both $\mathrm{Fe}_{3} \mathrm{O}_{4}$ and DCPEG- $\mathrm{Fe}_{3} \mathrm{O}_{4}$ MNPs show a superparamagnetic behavior at room temperature, with negligible hysteresis and remanence $\left(M_{\mathrm{R}}\left(\mathrm{Fe}_{3} \mathrm{O}_{4}\right)=1.19 \mathrm{emu} \mathrm{g}^{-1}\right.$ and $M_{\mathrm{R}}\left(\mathrm{DCPEG}_{10000}-\mathrm{Fe}_{3} \mathrm{O}_{4}\right)=$ 0.93 emu. $^{-1}$ )-Fig. 2. The saturation magnetization of the samples is 70.0 emu g $^{-1}$ (iron oxide) for the $\mathrm{Fe}_{3} \mathrm{O}_{4}$ and 67.9 emu.g $^{-1}$ for the DCPEG- $\mathrm{Fe}_{3} \mathrm{O}_{4}$. Thereby it becomes possible to conclude that the magnetite core remains intact and the magnetic properties were kept.

Stability of the PEG coated nanoparticles was tested by measuring the $\zeta$ potential through a range of temperatures. Fig. 3A shows that uncoated MNPs exhibit a negative potential whereas, when coated with DCPEG, the MNPs' potential increases with increasing Mw. In all cases no significant changes of $\zeta$ potential were noticed with temperature indicating that DCPEG offers a stable coating to the MNPs.

To study the effects of $\mathrm{pH}$ and ionic strength on colloidal stability, MNPs were exposed to a range of pHs from 2 to 12 and to different $\mathrm{NaCl}$ concentrations (from 0 to $1 \mathrm{M}$ ). Fig. 3B shows that the MNPs coated with $\mathrm{DCPEG}_{20000}$ are more stable in $\mathrm{pHs}$ ranging from 9-12 and 2-4, and the ones coated with DCPEG 5000 reveal more stability for basic pHs. The size distribution shown in Fig. 4 demonstrates that the MNPs size remains almost unchanged at $\mathrm{NaCl}$ concentrations as high as $1 \mathrm{M}$ proving that the influence of ionic strength on the colloidal stability can effectively be reduced

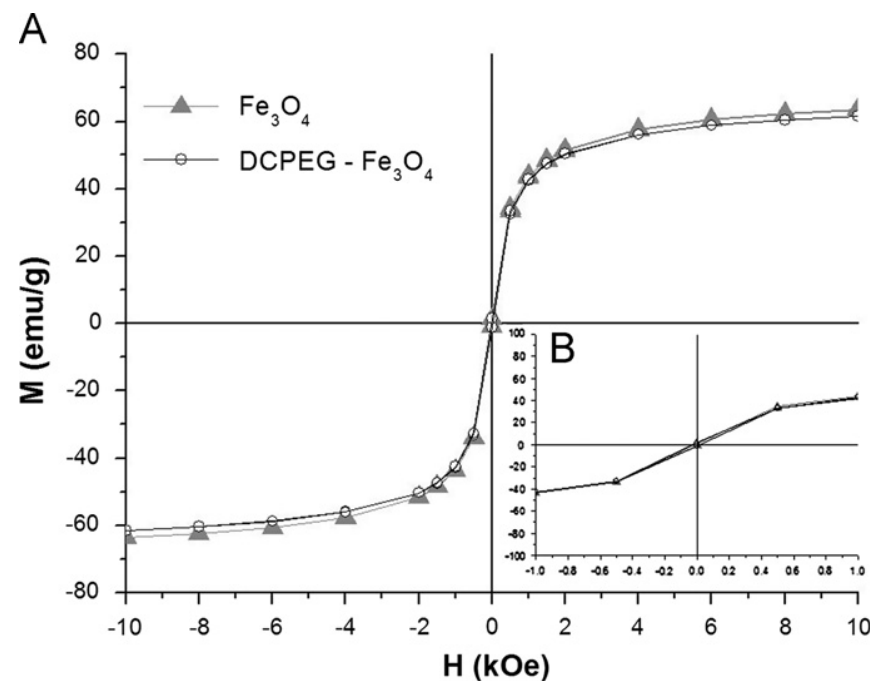

Fig. 2. Magnetization curves obtained by VSM at room temperature of $\mathrm{Fe}_{3} \mathrm{O}_{4}$ and DCPEG $_{10,000}-\mathrm{Fe}_{3} \mathrm{O}_{4}$ MNPs. (A) Scale from $-10 \mathrm{kOe}$ to $10 \mathrm{kOe}$ and (B) Scale from -1 kOe to 1 kOe.
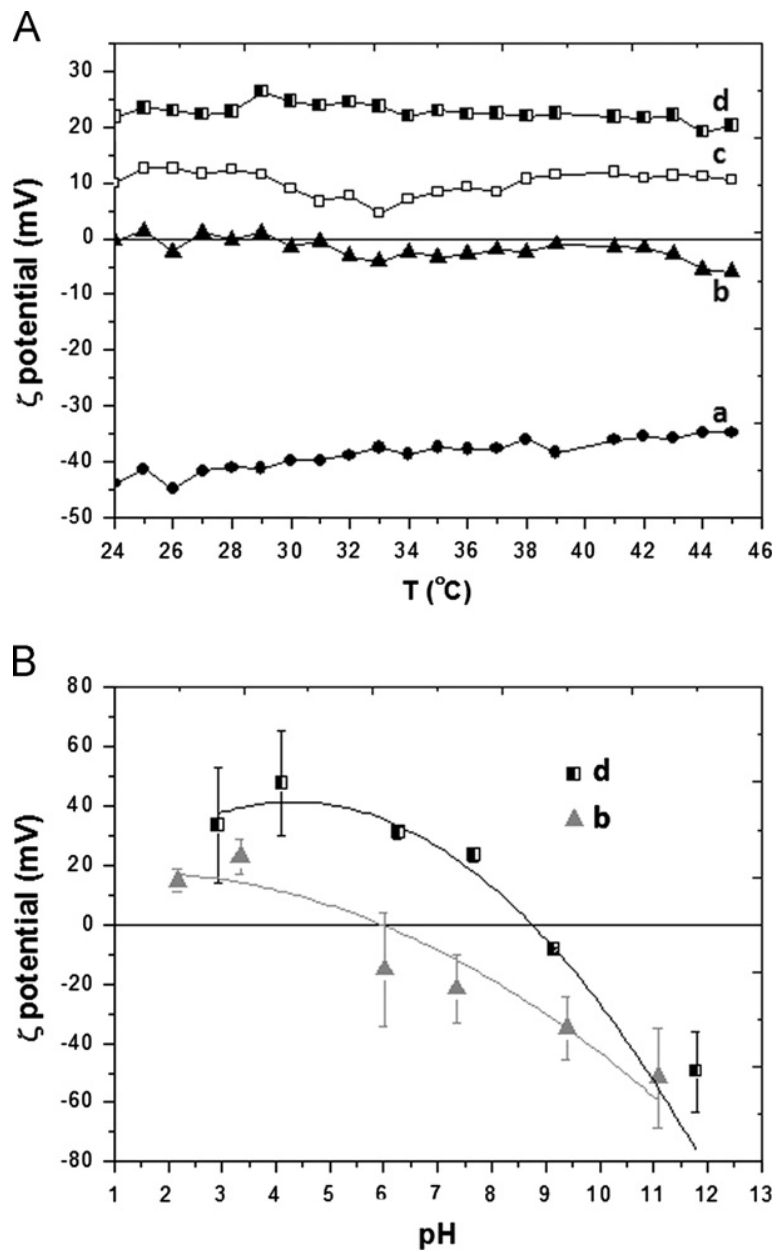

Fig. 3. Evolution of $\zeta$ potential measurements with $(\mathrm{A})$ temperature and $(\mathrm{B}) \mathrm{pH}$ (a: $\mathrm{Fe}_{3} \mathrm{O}_{4}$, b: $\mathrm{DCPEG}_{5000}-\mathrm{Fe}_{3} \mathrm{O}_{4}$, c: $\mathrm{DCPEG}_{10,000}-\mathrm{Fe}_{3} \mathrm{O}_{4}$, d: $\mathrm{DCPEG}_{20,000}-\mathrm{Fe}_{3} \mathrm{O}_{4}$ ).

with the $\mathrm{DCPEG}_{20000}$ coating. In the literature there are calculations that estimate the maximum chain length of $\mathrm{DCPEG}_{20000}$ as being $200 \mathrm{~nm}$ [19]. Thereby, in Fig. 4 it is observed that the distribution peak is always below $200 \mathrm{~nm}$, except for graphs B and $\mathrm{C}$, in which the distribution is not as narrower as for the other concentrations, and a second peak appears indicating that for $0.001 \mathrm{M}$ and $0.1 \mathrm{M}$ concentrations, some aggregation must be present. For the DCPEG 5000 (see supporting information) it was found that the MNPs were not stable for concentrations below $0.1 \mathrm{M}$.

\section{Conclusions}

In summary, biocompatible, water-soluble DCPEG-Fe $\mathrm{O}_{4} \mathrm{MNPs}$ of varying size were synthesized by surface modification of the $\mathrm{Fe}_{3} \mathrm{O}_{4}$ core by silanization reaction. MNPs colloidal stability was evaluated based on DLS and $\zeta$ potential measurements performed at room temperature. We concluded that DCPEG has an important effect on size, and charge properties of the MNPs. Among the different formulations tested, $\mathrm{DCPEG}_{20000}-\mathrm{Fe}_{3} \mathrm{O}_{4}$ MNPs kept the morphology of $\mathrm{Fe}_{3} \mathrm{O}_{4}$ core and showed colloidal stability, with non-detectable aggregation, when exposed to different salt concentration, $\mathrm{pH}$ and temperature. This, plus the multiple free carboxylic groups present on the nanoparticles surface makes DCPEG- $\mathrm{Fe}_{3} \mathrm{O}_{4}$ MNPs suitable for enhancing contrast in MRI and for drug delivery purposes. 

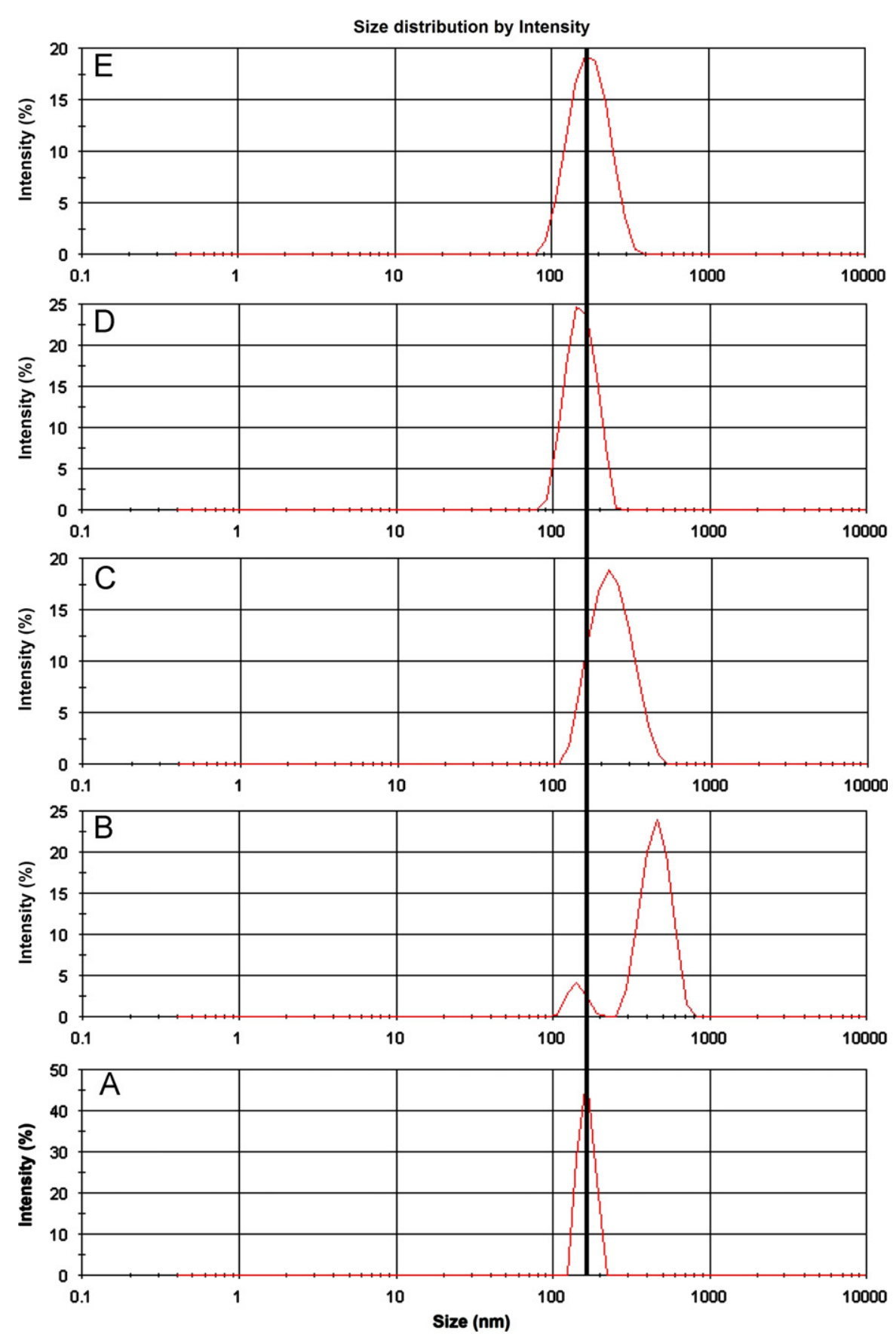

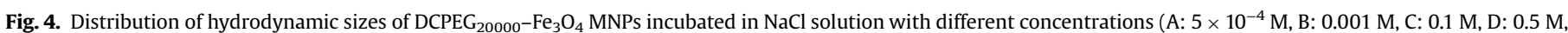
E: $1 \mathrm{M})$.

\section{Acknowledgments}

This work was supported by the Science and Technology Foundation (FCT) projects SUPRARELAX and PTDC/CTM-BPC/112774/2009. E. C. acknowledges the Health and Progress Foundation for their financial support through the Andalusian Initiative for Advanced Therapies (Andalusian Regional Ministry of Health).

\section{Appendix A. Supporting information}

Supplementary data associated with this article can be found in the online version at http://dx.doi.org/10.1016/j.matlet.2013.03.058.

\section{References}

[1] Qiao R, Yang C, Gao M. Superparamagnetic iron oxide nanoparticles: from preparations to in vivo MRI applications. J Mater Chem 2009;19(35):6274-93.

[2] Lefebure S, et al. Monodisperse magnetic nanoparticles: Preparation and dispersion in water and oils. J Mater Res 1998;13(10):2975-81.

[3] Bacri J-C, et al. Ionic ferrofluids: A crossing of chemistry and physics. J Magn Magn Mater 1990;85(1-3):27-32.

[4] Feldman K, et al. Probing resistance to protein adsorption of oligo(ethylene glycol)-terminated self-assembled monolayers by scanning force microscopy. J Am Chem Soc 1999;121(43):10134-41.

[5] Thanh NTK, Green LAW. Functionalisation of nanoparticles for biomedical applications. Nano Today 2010;5(3):213-30.

[6] Verma A, Stellacci F. Effect of surface properties on nanoparticle-cell interactions. Small 2010;6(1):12-21.

[7] Fang C, et al. Functionalized nanoparticles with long-term stability in biological media. Small 2009;5(14):1637-41. 
[8] Lee $\mathrm{H}$, et al. Antibiofouling polymer-coated superparamagnetic iron oxide nanoparticles as potential magnetic resonance contrast agents for in vivo cancer imaging. J Am Chem Soc 2006;128(22):7383-9.

[9] Li Z, et al. One-pot reaction to synthesize biocompatible magnetite nanoparticles. Adv Mater 2005;17(8):1001-5.

[10] Hu FQ et al. Preparation of biocompatible magnetite nanocrystals for in vivo magnetic resonance detection of cancer. Adv Mater 2006;18(19):2553-6.

[11] $\mathrm{Hu} \mathrm{F}$, et al. Preparation of magnetite nanocrystals with surface reactive moieties by one-pot reaction. J Colloid Interface Sci 2007;311(2):469-74.

[12] Jia $Q$ et al. Gelification: an effective measure for achieving differently sized biocompatible $\mathrm{Fe}_{3} \mathrm{O}_{4}$ nanocrystals through a single preparation recipe. J Am Chem Soc 2011;133(48):19512-23.

[13] Liu S, et al. Investigations on the interactions between plasma proteins and magnetic iron oxide nanoparticles with different surface modifications. J Phys Chem C 2010;114(49):21270-6.
[14] Cheng F-Y, et al. Characterization of aqueous dispersions of $\mathrm{Fe}_{3} \mathrm{O}_{4}$ nanoparticles and their biomedical applications. Biomaterials 2005;26(7):729-38.

[15] Qiao R, et al. Receptor-mediated delivery of magnetic nanoparticles across the blood-brain barrier. ACS Nano 2012;6(4):3304-10.

[16] Liu S, et al. A novel type of dual-modality molecular probe for $\mathrm{mr}$ and nuclear imaging of tumor: preparation, characterization and in vivo application. Mol Pharma 2009;6(4):1074-82.

[17] Feng B, et al. Synthesis of $\mathrm{Fe}_{3} \mathrm{O}_{4}$ /APTES/PEG diacid functionalized magnetic nanoparticles for MR imaging. Colloids Surf A: Physicochemical Eng Aspects 2008;328(1-3):52-9.

[18] Xie J, et al. Controlled PEGylation of monodisperse $\mathrm{Fe}_{3} \mathrm{O}_{4}$ nanoparticles for reduced non-specific uptake by macrophage cells. Adv Mater 2007;19 (20):3163-6.

[19] Choi CHJ, et al. Targeting kidney mesangium by nanoparticles of defined size. Proc Natl Acad Sci 2011;108(16):6656-61. 